Revista Colombiana de Obstetricia y Ginecología Vol. 55 No.4 • $2004 \cdot(308-314)$

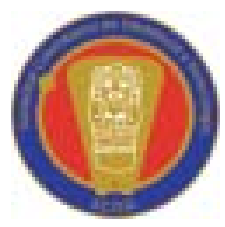

ARtículo De Revisión

\title{
ESTEROIDES ANTENATALES PARA MADURACIÓN PULMONAR. ¿CURSO ÚNICO O CURSOS MÚLTIPLES? UNA PREGUNTA AÚN VIGENTE.
}

\author{
WHICH REGIMEN OF ANTENATAL CORTICOSTEROIDS IS BETTER TO \\ IMPROVE LUING MATUIRITY, SINGLE OR MUILTIPLE COUIRSE? IT'S A \\ VALID QUIESTION TODAY.
}

Gladis Adriana Vélez, M.D.*

Recibido: agosto 27/2004 - Revisado: octubre 11/2004 - Aceptado: noviembre 22/2004

\section{RESUIMEN}

Se hace una revisión de la administración antenatal de esteroides para acelerar la maduración pulmonar fetal, su origen, bases fisiológicas y utilización en la práctica clínica a través del tiempo haciendo énfasis en el examen de los posibles beneficios y riesgos de la administración de cursos repetidos. Al sopesar la evidencia e incertidumbre alrededor de los riesgos de los cursos repetidos, no se recomienda su uso de rutina en la práctica clínica.

Palabras clave: dexametasona, betametasona, parto prematuro.

\section{SUMMARY}

An overview about the evolution of antenatal steroids administration in order to accelerate the lung maturity was done; it includes some topics about its origin, physiologic principles and use along the time. The main objective was to review the evidence of possible beneficial and harmful effects after multiple courses. Provided that concern exists, and some evidence about the possible risks

\footnotetext{
* Médica Especialista en Ginecología y Obstetricia. Aspirante a profesora auxiliar del Departamento de Obstetricia y Ginecología de la Universidad de Antioquia. Integrante del Centro Asociado al CLAP OPS/OMS Universidad de Antioquia. Correo electrónico: gadrianav@epm.net.co
}

of weekly courses, they should not be prescribed routinely for women at risk of preterm delivery.

Key Words: corticosteroids, respiratory distress syndrome, premature labour,

\section{INTRODUCCIÓN}

Una de las pocas intervenciones obstétricas bien establecidas para el tratamiento de la mujer embarazada en riesgo de tener un parto pretérmino, es la administración antenatal de esteroides para mejorar el resultado perinatal. Es quizás uno de los modelos más claros de la aplicación de la medicina basada en la evidencia: primero, a partir de la observación de un modelo animal, se estableció una hipótesis que pasó a ser probada en humanos; luego, múltiples ensayos clínicos corroboraron la utilidad de la medida y posteriormente éstos fueron sintetizados en metanálisis. Paradójicamente, es a la vez una muestra clara del lento devenir del conocimiento médico y de la dificultad de la colectivización del mismo, pues hoy, a más de treinta años de ese primer estudio aún no está dicho todo y todavía algunas pacientes que se beneficiarían de la administración de los esteroides no los reciben eficientemente.

Desde 1994, cuando los Institutos Nacionales de Salud de los Estados Unidos convocaron la Con- 
ferencia de Consenso acerca de los efectos de los corticosteroides para la maduración fetal sobre el resultado perinatal, quedó claro que la administración de un curso único de esteroides reduce los riesgos de muerte neonatal, síndrome de dificultad respiratoria y hemorragia intra ventricular en los bebés prematuros y que este beneficio es máximo en los siete días siguientes. ${ }^{1}$ El tratamiento recomendado consiste en betametasona $12 \mathrm{mg}$ intramuscular cada 24 horas por 2 dosis o dexametasona 6 mg intramuscular cada 12 horas por 4 dosis.

El panel también concluyó que los beneficios y riesgos potenciales de la administración repetida luego del curso inicial, eran aún desconocidos y llamó la atención sobre la necesidad de investigación en ese sentido.

En el año 2000 y luego de más de cinco años de estas recomendaciones se observaba un fenómeno llamativo: si bien la utilización de los esteroides antenatales había tenido un aumento continuo, aún no alcanzaba las cifras esperadas, aunque pasó de aproximadamente 20\% en 1994 a $72 \%$ en el año $2000 .^{2}$ La práctica de administrar cursos repetidos había llegado a ser de uso corriente en Estados Unidos, Reino Unido y Australia. Tales cursos incluían dosis semanales, ocasionales o un curso de «rescate», definido como un curso único repetido, administrado inmediatamente antes del parto planeado o inminente.

En 1996 en una encuesta realizada a especialistas en medicina materno fetal en Estados Unidos, el 96\% afirmó que prescribía más de un curso de esteroides y el 58\% que prescribía seis o más. En 1999 en el Reino Unido el 98\% de los obstetras indicó que prescribía cursos repetidos ${ }^{3}$ y hubo reportes de casos en los cuales las madres recibieron hasta 16 cursos de esteroides antenatales. ${ }^{4}$ Esto motivó una nueva conferencia de Consenso cuyo objetivo era proporcionar al personal de salud, a las pacientes y al público en general una evaluación responsable de la evidencia disponible en ese momento acerca de los beneficios y riesgos de los cursos repetidos de esteroides antenatales. ${ }^{5}$ Las preguntas planteadas fueron:
En primer lugar, ¿la evidencia sobre los beneficios y riesgos de los cursos repetidos de esteroides antenatales era suficiente para permitir recomendaciones de consenso?

Segundo, si era así, ¿cuáles serían esas recomendaciones? Y por último, si no lo era, ¿qué información adicional se debería obtener?

Las conclusiones fueron: los datos disponibles eran inadecuados para argumentar a favor o en contra del uso de cursos repetidos o de rescate de esteroides antenatales para maduración pulmonar y por lo tanto se deberían reservar para pacientes incluidas en estudios aleatorios controlados.

Ellos recomendaron la realización de estudios clínicos aleatorios bien diseñados con el suficiente poder para evaluar la seguridad y eficacia, minimizando la exposición de la madre y el feto a los posibles riesgos. A pesar de estas recomendaciones, es frecuente en nuestro medio que aún se siga utilizando la administración de cursos repetidos semanalmente hasta la semana 34, e incluso esta es la pauta dada en algunas guías de manejo. ${ }^{6}$

\section{MECANISMOS DE ACCIÓN Y EFECTO DE LOS ESTEROIDES SOBRE EL PULMÓN FETAL}

Las primeras observaciones acerca de la influencia hormonal en la maduración de los órganos, se remontan a 1953 cuando Moog estudió el efecto del sistema hipófisis adrenal en la diferenciación de la fosfatasa en el duodeno de ratones lactantes. Luego Buckingham y colegas (1968) consideraron que el desarrollo del pulmón podría ser análogo al desarrollo del intestino.

Estas observaciones fueron ignoradas en su mayor parte hasta 1969 cuando Liggins, obstetra de Nueva Zelanda, estudiando la influencia de los glucocorticoides en el momento del parto en corderos, observó que algunos de los que habían recibido glucocorticoides eran viables a una edad más temprana de lo que podría esperarse. Esto lo llevó a suponer la posible influencia de los glucocorticoides en la aceleración de la maduración 
pulmonar y a investigar en esa dirección hasta iniciar en 1969 el primer ensayo clínico controlado, el cual se extendió hasta 1977 y aún hoy es el estudio más grande que se haya realizado acerca de este tema. ${ }^{7}$

Los glucocorticoides administrados durante el embarazo se unen a las proteínas transportadoras en la circulación materna dándose una distribución entre la circulación materna y fetal que es compleja e involucra un equilibrio entre las formas libre y unida. La betametasona y la dexametasona se unen menos que el cortisol a las globulinas y además la enzima 11 Beta-hidroxiesteroide-deshidrogenasa, presente en la placenta y que tiene la función de limitar el acceso de los esteroides maternos al feto inactivándolos, tiene poca afinidad por éstas. Solamente la forma libre ingresa a la célula y se une a un receptor específico. Este complejo glucocorticoide receptor activado se une a los elementos de respuesta a los glucocorticoides localizados a lo largo del genoma, luego de lo cual se incrementa la trascripción de genes específicos y el ARN mensajero resultante es transcrito en proteínas específicas.

La presencia o ausencia de estos receptores determina que un tejido en particular responda o no al estímulo del esteroide y su número limita la concentración hormonal máxima a medida que los receptores son copados.

El aumento en la velocidad de trascripción se inicia aproximadamente una hora después de la administración del esteroide y el máximo de incremento en el contenido de ARNm y proteínas se da entre 24 y 48 horas respectivamente. ${ }^{8}$ Esto explica por qué el beneficio es máximo si el parto se da después de 48 horas de la primera dosis de esteroide. Los regímenes recomendados usualmente conllevan una ocupación aproximada del $75 \%$ de los receptores disponibles, lo que produce una respuesta fetal en los órganos blancos cerca del máximo y por lo tanto no se justifican dosis más altas o más frecuentes.

Los esteroides causan citodiferenciación y cambios precoces en las proteínas responsables del desa- rrollo en por lo menos doce tejidos diferentes. Aceleran el ritmo de maduración sin alterar la secuencia. Tienen efecto en el hígado, sistema gastrointestinal, páncreas, piel, riñón y cerebro.

En el pulmón tienen un doble efecto, pues no sólo aumentan el surfactante sino que producen cambios estructurales tanto en las células epiteliales de la vía aérea como en los fibroblastos. Parece que estos cambios no revierten una vez ha pasado el tiempo de acción del esteroide, y esto podría explicar que el efecto protector persista, aunque en menor grado, mas allá de los siete días.

Los efectos de los glucocorticoides en el pulmón en desarrollo son:

- Incrementan el surfactante alveolar y tisular.

- Aumentan la distensibilidad y el volumen pulmonar máximo.

- Disminuyen la permeabilidad vascular.

- Aumentan el aclaramiento del líquido del pulmón.

- Aumentan la respuesta al surfactante.

¿ESTÁ RESUELTA LA CONTROVERSIA ACERCA DE LA UTILIZACIÓN DE UN CURSO ÚNICO O CURSOS MÚLTIPLES DE ESTEROIDES ANTENATALES?

Existe mucha incertidumbre acerca de por cuánto tiempo debe continuarse el tratamiento si la paciente permanece en riesgo de parto pretérmino después de siete días del curso inicial. La conferencia de Consenso del año 2000, como se dijo anteriormente, enfatizó en la necesidad de realizar estudios para aclarar este aspecto, debido principalmente a la inquietud que generan los informes de efectos adversos de los cursos múltiples, tanto en animales como en humanos.

Walfisch y colaboradores ${ }^{4}$ realizaron una revisión de estudios prospectivos bien diseñados tanto en animales como en humanos publicados en inglés desde 1966 hasta mayo de 2000. Encontraron 12 estudios con estas características en animales. No identificaron ensayos clínicos bien diseñados en humanos. 
En dos estudios aleatorios, controlados con placebo efectuados en corderos, se mostró una mejoría secuencial en la función pulmonar con 2, 3 y 4 dosis de betametasona. Sin embargo encontraron una restricción del crecimiento, claramente dosis - dependiente, que llegó hasta el 27\%.

Otros resultados que se han descrito en modelos animales con la administración de cursos repetidos de esteroides incluyen: disminución persistente del peso del pulmón fetal, aumento de la presión arterial con un incremento de la actividad adrenocortical, disminución significativa del tamaño cerebral, alteraciones en los receptores de esteroides en el hipocampo y degeneración de éste..$^{9-11}$

Obviamente, estas observaciones deben tomarse siempre con cautela, pues las especies son muy diferentes en cuanto a la duración de la gestación y al desarrollo cerebral. Sin embargo no se debe olvidar que fue de un modelo animal de donde surgió esta práctica clínica.

En humanos, los datos acerca de los riesgos y beneficios de los cursos múltiples de esteroides son extraídos de reportes de casos, estudios retrospectivos y estudios observacionales prospectivos. Se han descrito: cardiomiopatía hipertrófica transitoria, síndrome cushinoide neonatal, reducción del perímetro cefálico al nacimiento, aumento de la infección neonatal, bajo peso al nacer, aumento de la morbilidad infecciosa en la madre, insuficiencia adrenal materna y diabetes gestacional.

Existen varios metanálisis posteriores al año 2000 ninguno de los cuales ha dado una respuesta definitiva. El realizado por Aghajafari y colaboradores ${ }^{12}$ incluyó ocho artículos extraídos de las bases de datos Medline (1966 a 1999) y Embase (1980 a 1999). Sus criterios de inclusión fueron artículos completos, en inglés, que compararan un curso único con cursos múltiples y que el resultado medido fuera morbi - mortalidad materna, perinatal o infantil. Excluyeron los artículos que no controlaron las diferencias en la edad gestacional al momento del nacimiento. Los autores encontraron: una disminución significativa del riesgo de síndrome de dificultad respiratoria (OR 0,79
IC95\% 0,64 - 0,98); pero también una tendencia hacia el incremento del riesgo de displasia bronco pulmonar (OR 1,30 IC95\% 0,96 - 1,78) en el grupo de cursos múltiples. En cuanto al riesgo de hemorragia intraventricular, enterocolitis necrotizante, sepsis y muerte neonatal no hubo diferencia significativa.

En cuanto al riesgo de disminución del peso para la edad gestacional al nacimiento, no hubo diferencia entre los dos grupos. Cuatro estudios evaluaron el resultado materno. El riesgo de amnionitis no fue significativamente mayor (OR 1,46 IC95\% 0,47 - 4,59). El riesgo de endometritis posparto fue mayor en el grupo de cursos múltiples (OR 3,22 IC95\% 1,9 - 5,45).

Una de las mayores limitaciones de estos estudios es el sesgo de selección, ya que las pacientes no fueron asignadas en forma aleatoria y la variable más importante que debería haber sido controlada que era la edad gestacional al momento del curso inicial, solamente fue informada en un estudio.

En varias de estas revisiones se hace referencia a estudios clínicos aleatorios, que estaban en proceso desde el año 2000. ${ }^{13}$ Sin embargo, solamente se logró obtener los resultados de dos estudios clínicos aleatorios, publicados en inglés.

El primero, realizado por McEvoy y colaboradores y publicado en Pediatrics en agosto del 2002, ${ }^{14}$ es un ensayo clínico, doble ciego, en el cual 37 pacientes, luego de recibir el primer curso de esteroides, fueron asignadas aleatoriamente a recibir cursos semanales de corticosteroides o de placebo, hasta la semana 34 o hasta el parto. Los resultados medidos fueron la capacidad funcional residual y la adaptabilidad respiratoria medidas en las primeras 48 horas posparto, y como resultados secundarios, algunos parámetros clínicos como el perímetro cefálico, la administración de surfactante y los días que los neonatos requirieron oxígeno o ventilación mecánica. No hubo diferencia estadísticamente significativa para ninguno de los resultados y aunque se hubiese presentado, las implicaciones de la misma serían de difícil interpretación, pues el tamaño de la muestra no tenía 
el poder para evaluar resultados clínicos ya que fue calculado con base en los cambios en la capacidad residual funcional como resultado primario.

El segundo estudio ${ }^{15}$ es un trabajo multicéntrico (13 hospitales de Estados Unidos) de Guinn y colaboradores, que se llevó a cabo entre febrero de 1996 y abril del 2000. Las pacientes elegibles fueron aquellas en alto riesgo de parto pretérmino entre 24 y $32+6$ semanas, bien fuera por trabajo de parto pretérmino, ruptura prematura de membranas ovulares o por indicaciones materno fetales de terminar la gestación. Se excluyeron aquellas pacientes que tenían necesidad de parto inmediato; anomalías fetales incompatibles con la vida, madurez pulmonar documentada, tuberculosis materna activa e infección por VIH. Todas las pacientes recibieron un curso inicial de esteroides y aquellas que siete días después no habían tenido su parto, fueron asignadas por un proceso de aleatorización simple, a recibir $12 \mathrm{mg}$ intramuscular de betametasona o un placebo cada 24 horas por dos dosis cada semana hasta el parto.

Ellos determinaron un tamaño de muestra de 1.000 pacientes para tener un poder del 90\% para detectar una reducción de un tercio en un resultado compuesto, definido como la presencia de cualquiera de las siguientes complicaciones: síndrome de dificultad respiratoria severo, displasia bronco pulmonar, hemorragia intra ventricular severa, leucomalacia periventricular, enterocolitis necrotizante, sepsis probada o muerte entre el ingreso al estudio y el alta luego del parto.

En 1999 realizaron un análisis interino que se había programado desde el inicio. En ese momento habían ingresado 308 pacientes; 161 en el grupo de tratamiento y 147 en el del placebo. Evaluaron la morbilidad compuesta y no encontraron una diferencia importante. Debido a esto, y a informes aparecidos en esa época acerca de los riesgos potenciales de los esteroides, realizaron un análisis de poder condicional y determinaron que si la tendencia continuaba como hasta el momento, la probabilidad de encontrar una diferencia significativa con las 1.000 pacientes era menor del 2\% y decidieron terminar el estudio con 502 pacientes, 256 en el grupo de cursos repetidos de esteroides y 246 en el grupo placebo.

No hubo diferencias en los aspectos demográficos de las pacientes al inicio y no hubo diferencia significativa en la disminución del resultado compuesto $(28 \%$ en los neonatos del grupo de curso único y 22,5\% en los del grupo de cursos semanales, $\mathrm{p}=0,16$ )

Realizaron un análisis estratificado por edad gestacional para evaluar si algún subgrupo específico se había beneficiado de los cursos semanales. En todos estos análisis de subgrupos, la morbilidad compuesta fue menor en el grupo de cursos semanales pero solamente fue estadísticamente significativa en el grupo que tuvo el parto antes de las 28 semanas.

En un análisis secundario individual de resultados, hubo una disminución significativa del síndrome de dificultad respiratoria severo en el grupo de cursos semanales comparado con el grupo placebo $(15,3 \%$ vs $24,1 \%$, riesgo relativo de 0,63 con un intervalo de confianza del $95 \%$ de 0,44 a 0,93 ), pero esto no se acompañó de un aumento en la sobrevida o de una reducción de la displasia bronco pulmonar. Los autores justifican su decisión de suspender el estudio por el temor a los efectos adversos, dado que hubo un incremento en la incidencia de la hemorragia intra ventricular severa en los neonatos expuestos a los cursos repetidos. Concluyeron que dada la aparente falta de eficacia y los riesgos potenciales a corto y largo plazo, los cursos repetidos de esteroides no deben utilizarse rutinariamente.

Obviamente las críticas a este trabajo no se hicieron esperar y el primer argumento en contra es la reducción del tamaño de muestra a mitad del estudio que rebajó el poder del 90\% al 27\%. En JAMA fueron publicadas tres cartas al editor ${ }^{16}$ haciendo referencia al tema. En una de ellas los autores hacen una interpretación diferente de los resultados de Guinn. Sostienen que fue un error la terminación prematura del estudio y resaltan la disminución del síndrome de dificultad respiratoria en el grupo de 24 a 27 semanas, como un 
hallazgo importante, a la vez que minimizan el resultado del aumento de la hemorragia intraventricular en el grupo de cursos múltiples (ambos fueron análisis de subgrupos y por lo tanto tienen el mismo valor limitado) y argumentan a favor de la continuación de los estudios clínicos en proceso, uno de ellos en Canadá, coordinado por la doctora Kelly Murphy (coautora de esta carta). Este es el conocido como protocolo MACS $^{17}$ por sus siglas en inglés, del cual están haciendo parte tres centros en nuestro país y el cual pretende incluir 1.900 pacientes con seguimiento hasta los 18 y 24 meses.

La revisión sobre este tema, de la Colaboración Cochrane, ${ }^{18}$ se hizo sobre un total de 551 pacientes incluidas en los tres estudios ya mencionados: 502 pacientes del trabajo de Guinn y colaboradores, 37 del de McEvoy y col. y 12 pacientes de un estudio piloto del MACS. Los tres trabajos fueron calificados como de alta calidad y por lo tanto no fueron sometidos a análisis de sensibilidad. Afirman que hubo evidencia de una reducción en el síndrome de dificultad respiratoria grave, resultado dado exclusivamente por el trabajo de Guinn y que se debe mirar con precaución por lo expuesto anteriormente. En concordancia con este resultado, también en el estudio de Guinn, un número menor de neonatos requirieron surfactante en el grupo de cursos múltiples comparado con el de curso único (40 casos vs 59, $\mathrm{p}=0,01)$. Aunque en la revisión de Cochrane dicen que el trabajo de McEvoy también apoyó este último resultado, en el artículo publicado en Pediatrics los autores afirman claramente que no hubo ninguna diferencia significativa entre los dos grupos en cuanto a los resultados clínicos. Sin embargo, la conclusión práctica es que no existe evidencia suficiente sobre los riesgos y beneficios para recomendar la administración de cursos repetidos de esteroides para las mujeres a riesgo de parto pretérmino.

En un futuro más cercano, esperamos la publicación de los resultados de dos trabajos terminados en diciembre de 2003, uno del Reino Unido con 154 pacientes y otro de los Institutos Nacionales de la salud de los Estados Unidos, con 492. Igual- mente está en proceso otro estudio colaborativo entre Australia y Nueva Zelanda con 980 pacientes.

Siendo optimistas diríamos que sumando estos trabajos se tendrá un tamaño de muestra suficiente para responder con certeza esta pregunta; sin embargo la validez de los resultados depende también de muchas otras variables, que habrá que considerar en el momento de publicación de los resultados.

Un solo ciclo de dos dosis de esteroides disminuye el riesgo de muerte neonatal en un 39\% y el de síndrome de dificultad respiratoria en un 49\%, ${ }^{19}$ con la tranquilidad de no tener efectos adversos a corto ni a largo plazo, con seguimiento hasta por 20 años. ${ }^{20}$ La pregunta fundamental es si es posible mejorar estos resultados: ¿al aumentar el número de ciclos se obtendrán más beneficios sin aumentar los efectos adversos? El análisis de la información que poseemos hasta ahora, apunta hacia una respuesta negativa.

En conclusión, si bien los datos actuales son aún insuficientes, la evidencia está más en contra que a favor de los cursos múltiples y por lo tanto no deben utilizarse fuera del contexto de estudios clínicos, permaneciendo atentos obviamente a los resultados de los estudios mencionados.

\section{REFERENCIAS}

1. National Institutes of Health. N.I.H. Report of the Consensus Development Conference on the Effect of Corticosteroids for Fetal Maturation on Perinatal outcome. Publication No. 95-3784. Bethesda, MD: National Institute Of Child Health and Human Development; 1994.

2. Meadow WL, Bell A, Sunstein CR. Statistics, not memories: what was the standard of care for administering antenatal steroids to women in preterm labor between 1985 and 2000? Obstet Gynecol 2003;102:356-62.

3. Brocklehurst P, Gates S, Mckenzie-McHarg K, Alfirevic Z, Chamberlain G. Are we prescribing multiple courses of antenatal corticosteroids? A survey of practice in the UK. Br J Obstet Gynaecol 1999;106:977-9.

4. Walfisch A, Haalak M, Mozer M. Multiple courses of antenatal steroids: risks and benefits. Obstet Gynecol 2001;98:491-7. 
5. National Institutes of Health Consensus Development Panel. Antenatal corticosteroides revisited: repeat courses - National Institutes Of Health Consensus Development Conference Statement, August 17-18, 2000. Obstet Gynecol 2001;98:144-50.

6. Jubiz A. Guías de Atención Materna y Perinatal con enfoque de Riesgo. Dirección Seccional de Salud de Antioquia; 2001.

7. Liggins GC, Howie RN. A controlled trial of antepartum glucocorticoid treatment for prevention of the respiratory distress syndrome in premature infants. Pediatrics 1972;50:515-25.

8. Ballard PL, Ballard RA. Scientific basis and therapeutic regimens for use of antenatal glucocorticoids. Am J Obstet Gynecol 1995;173:254-62.

9. Huang WL, Beazley LD, Quinlivan JA, Evans SF, Newnham JP, Dunlop SA. Effect of corticosteroids on brain growth in fetal sheep. Obstet Gynecol 1999;94:213-8.

10.Edwards HE, Burnham WM. The impact of corticosteroids on the developing animal. Pediatr Res 2001;50:433-40.

11.Aghajafari F, Murphy K, Matthews S, Ohlsson A, Amankwah, Hannah M. Repeated doses of antenatal corticosteroids in animals: a systematic review. Am J Obstet Gynecol 2002;186:843-9.

12.Aghajafari F, Murphy K, Willan A, Ohlsson A, Amankwah K, Matthews S, et al. Multiple courses of antenatal corticosteroides: a systematic review and metaanalysis. Am J Obstet Gynecol 2001;185:1073-80.
13. Leung TN, Lam PM, Ng PC, Lau TK. Repeated courses of antenatal corticosteroides: is it justified? Acta Obstet Gynecol Scand 2003;82:589-96.

14.McEvoy C, Bowling S, Williamson K, Lozano D, Tolaymat L,Izquierdo 1 , et al. The effect of a single remote course versus weekly courses on functional residual capacity In preterm infant: a randomized trial. Pediatrics 2002;110: 280-4.

15. Guinn DA, Atkinson MW, Sullivan L, Lee MJ, MacGregor S, Parilla BV, et al. Single vs weekly courses of antenatal corticosteroids for women at risk of preterm delivery: a randomized controlled trial. JAMA 2001;286:1581-7.

16. Murphy KE, Hannah M, Brocklehurst P. Are weekly courses of antenatal steroids beneficial or dangerous? JAMA 2002;287:188.

17.Aghajafari F, Murphy K, Ohlsson A, Amankwah K, Matthews S, Hannah ME. Multiple versus single courses of antenatal corticosteroids for preterm birth: a pilot study. J Obstet Gynaecol Can 2002;24:321-9.

18. Crowther CA, Harding J. Repeat doses of prenatal corticosteroids for women at risk of preterm birth for preventing neonatal respiratory disease (Cochrane review). In: The Cochrane Library, Issue 4, 2003.

19. Crowley P, Chalmers I, Keirse MJ. The effects of corticosteroid administration before preterm delivery: an overview of the evidence from controlled trials. Br J Obstet Gynaecol 1990;97:11-25.

20.Dessens AB, Haas H, Koppe JG. Twenty-year followup of antenatal corticosteroid treatment. Pediatrics 2000;105:E77. 